\title{
ASPECTOS REPRESENTACIONAIS EM QUESTÕES DO ENEM NA ÁREA DE CIÊNCIAS DA NATUREZA
}

\author{
REPRESENTATIONAL ASPECTS IN ENEM ISSUES AROUND NATURAL SCIENCES
}

Robson Fagner Ramos de Araújo ${ }^{1}$, Ulisses Vieira Guimarães², Edson José Wartha ${ }^{3}$

Recebido: junho/2020 Aprovado: outubro/2020

\begin{abstract}
Resumo: Considerando a importância das representações usadas como suporte em questões do Exame Nacional do Ensino Médio, esta pesquisa objetiva verificar se os aspectos representacionais e textuais, presentes como suporte nas questões na área de Ciências da Natureza, são determinantes no desempenho dos estudantes. Para tanto, o estudo foi realizado por meio de análise estatística descritiva do desempenho em questões previamente selecionadas dos exames realizados nos anos de 2012, 2013 e 2014. Os resultados indicam que os aspectos representacionais e textuais não se configuram como determinantes no desempenho escolar. Nas questões selecionadas para análise e no cruzamento com os dados socioeconômicos verificamos que o fator determinante do desempenho escolar é o fator renda familiar.
\end{abstract}

Palavras-chave: desempenho escolar; aspectos representacionais; aspectos textuais.

\begin{abstract}
Considering the importance of the representations used as support in questions of the National Examination of High School, this research aims to verify if the representational and textual aspects, present as support in the questions in the area of Sciences of the Nature, are determinant in the performance of the students. To do so, the study was performed through a descriptive statistical analysis of the performance in questions previously selected from the examinations carried out in the years of 2012, 2013 and 2014. The results indicate that the representational and textual aspects do not constitute determinants in school performance. In the questions selected for analysis and in the cross-referencing with socioeconomic data, we verified that the determinant of school performance is the family income factor.
\end{abstract}

Keywords: school performance; representational aspects; textual aspects.

\section{Introdução}

O Exame Nacional do Ensino Médio (ENEM) tem como objetivo principal avaliar os concluintes e egressos do Ensino Médio. O exame é realizado anualmente desde 1998 e, após as primeiras edições, passou também a ser utilizado como parte da nota em processos seletivos para cursos de graduação em instituições de Ensino Superior. Em 2009, o ENEM teve modificações significativas, sendo que a principal modificação diz respeito à sua nova função, ou seja, substituir processos seletivos de admissão na graduação em algumas instituições de ensino federais do país. Nos últimos exames realizados, verificou-se que a maioria das instituições de Ensino Superior usam total ou parcialmente as notas do ENEM em seus processos

\footnotetext{
1 iD https://orcid.org/0000-0002-7155-6706 - Mestre em Ensino de Ciências e Matemática pela Universidade Federal de Sergipe (UFS). Docente na ECITE Arlinda Pessoa da Silva, Juru, Paraíba, Brasil. Trav. Padre Cícero, no 114, centro, Juru - PB, Brasil. E-mail: robson.ramos.araujo@hotmail.com

2 iD https://orcid.org/0000-0003-4203-8640- Doutor em Educação Matemática pela Universidade Anhanguera de São Paulo. Docente (UFS), São Cristóvão, Sergipe, Brasil (Av. Marechal Rondon, s/n, DECAT/CCET, 49100-000, São Cristóvão, Sergipe, Brasil). EMail: prof.ulisses.ufs@gmail.com

3 iD https://orcid.org/0000-0003-4919-3504-Doutor em Ensino de Ciências (USP) na qual o diploma foi obtido. Professor Associado da Universidade Federal de Sergipe (UFS), São Cristóvão, Sergipe, Brasil. Cidade Univ. Prof. José Aloísio de Campos, Av. Marechal Rondon, s/n, Jd. Rosa Elze, São Cristóvão/SE/Brasil, CEP 49100-000. E-mail: ejwartha@academico.ufs.br
} 
seletivos. O ENEM é um exame realizado em média por seis milhões de estudantes todos os anos.

A partir de 2009, o ENEM passou a ser aplicado em dois dias e está estruturado por meio de quatro matrizes, uma para cada área de conhecimento. A área de Ciências da Natureza e suas Tecnologias, objeto deste estudo, é composta por 45 questões, que resultam em uma avaliação de 180 itens de múltipla escolha, que avaliam diferentes competências e habilidades apresentadas na Matriz de Referência para o ENEM, além de uma redação (BRASILa, 2009).

O ENEM procura apresentar uma prova interdisciplinar e contextualizada, com a intenção de "verificar" competências e habilidades (BRASILb, 2009). Nesse contexto, as definições de competências e habilidades são entendidas como:

Competências são as modalidades estruturais da inteligência, ou melhor, ações e operações que utilizamos para estabelecer relações com e entre objetos, situações, fenômenos $e$ pessoas que desejamos conhecer. As habilidades decorrem das competências adquiridas e referem-se ao plano imediato do 'saber fazer'. Por meio das ações e operações, as habilidades aperfeiçoam-se e articulam-se, possibilitando nova reorganização das competências (BRASIL, 1999, p. 7).

Entretanto, o sucesso efetivo deste sistema de avaliação em larga escala depende de que as provas do ENEM sejam bem formuladas, apresentando questões consistentes com a devida avaliação das habilidades e competências preconizadas para o Ensino Médio, garantindo a que o exame também exerça a função de um auxiliador da escola para que se construa o conhecimento do aluno, "desenvolvendo capacidades de aprender, criar, formular, ao invés do simples exercício de memorização" (BRASIL, 2000, p. 5).

A partir de 2009, as questões de múltipla escolha passaram a ser estruturadas em quatro matrizes, uma para cada área de conhecimento (linguagens, códigos e suas tecnologias (incluindo redação); ciências humanas e suas tecnologias; ciências da natureza e suas tecnologias e matemática e suas tecnologias), sendo que cada uma das áreas se compõe de quarenta e cinco questões. Assim, o exame passou a ser dividido em dois cadernos, aplicado em dois dias e contendo, ainda, a proposta de produção de texto. Nesse estudo, focamos nas capacidades dos estudantes em analisar e responder questões na área de ciências da natureza e suas tecnologias considerando habilidades que ressaltam aspectos representacionais na resolução dos itens, mais especificadamente em relação as habilidades descritas a seguir:

1) H17- relacionar informações apresentadas em diferentes formas de linguagem e representações usadas em química, bem como nos textos discursivos, gráficos, tabelas, relações matemáticas e na linguagem simbólica que estão envolvidos o conhecimento científico;

2) H24- Utilizar códigos e nomenclatura da química para caracterizar materiais, substâncias ou transformações químicas;

3) H25-Caracterizar materiais ou substâncias, identificando etapas, rendimentos ou implicações biológicas, sociais, econômicas ou ambientais de sua obtenção ou produção (BRASIL, 2009, p 89). 
A partir do referencial teórico-metodológico do ENEM, é possível inferir que o exame nacional se baseia em alguns postos-chaves, tais como: competências/habilidades, foco na cidadania, contextualização, interdisciplinaridade e utilização de situações-problema. Nessa perspectiva, tais aspectos devem ser compreendidos em conjunto com o propósito do desenvolvimento dos objetivos do contexto educacional como um todo. Contudo, como salientam Silva e Ribas (2003), muitos professoresapresentam dificuldades em inserir esses aspectos em suas aulas ou não as consideram importante o desenvolvimento de habilidades relacionadas aos aspectos representacionais do conhecimento científico.

Vale destacar que a partir do ano de 2003, o ENEM realizou uma mudança importante ao inserir na prova um questionário socioeconômico, que é respondido no momento da inscrição no exame, com a finalidade de conhecer melhor o perfil dos participantes. Essas informações podem auxiliar a conhecer melhor o perfil dos estudantes que realizam o exame e as condições de estudo durante toda a Educação Básica.

Este artigo faz parte de uma dissertação de mestrado (ARAúJO, 2017) inserida em um projeto de pesquisa mais amplo intitulado: "Aspectos representacionais e textuais na área de ciências da natureza: um olhar sobre os itens do ENEM", sendo financiando pela FAPITEC (Fundação de Amparo à Pesquisa e à Inovação Tecnológica do Estado de Sergipe ). Neste sentido, buscamos identificar se os aspectos representacionais e textuais são determinantes no desempenho dos estudantes na área Ciências da Natureza com participantes do Estado de Sergipe que realizaram o ENEM nos anos de 2012, 2013 e 2014. Buscamos, também, identificar se fatores relacionados à heterogeneidade dos participantes como o contexto social, cultural, econômico, familiar, dentre outros, influenciam no desempenho deles no Exame.

\section{A Prática de Leitura de Texto Científico na Compreensão dos Aspectos Representacionais no Ensino de Ciências}

A constituição histórica da leitura no âmbito escolar é marcada pela decodificação da palavra, uma vez que, geralmente, estão embasadas em respostas prontas e rápidas dos questionamentos estabelecidos previamente, sendo considerados repetições formais. Dessa forma, questões relacionadas às capacidades leitoras dos estudantes podem provocar obstáculos na interação pessoal dos leitores, seja com o texto ou com as ideias construídas pelos estudantes. A capacidade leitora está ligada à capacidade de se interpretar, atribuir sentido e alcançar a clareza sobre as informações que aquele conjunto de ideias apresenta ao interlocutor. Essa compreensão é fundamental para a construção de conhecimento por parte dos estudantes, pois o aprendizado só é efetivo quando o leitor é capaz de compreender os textos debatidos, que, na maioria das vezes, não ocorre de maneira direta, mas a leitura passa a ser um processo que precisa ser aprendido (MARQUES, 2001).

Nesse contexto, os processos de ler e escrever tornam-se essenciais e devem ser trabalhados nas aulas de Ciências, auxiliando os leitores na construção de conhecimentos científicos. Para Francisco Junior et al. (2010), os estudantes apresentam dificuldades de interpretar questões e problemas que estão inseridos nos conteúdos de Ciências. Wenzel (2013) enfatiza que a prática da leitura possibilita ao estudante contato com uma nova linguagem, mais 
específica, e, por isso, a leitura em sala de aula precisa ser orientada. Para Granger (1974), o conhecimento científico se desdobra num universo de linguagem e Lemke (1997) esclarece que, para aprender a linguagem da ciência, torna-se indispensável "Aprender Ciências". E a aprendizagem em ciências implica aprender a falar o idioma próprio das Ciências, uma vez que tem o seu próprio e exclusivo modelo semântico e suas próprias formas de construir significados, ou seja, também é necessário compreender que as representações fazem parte do processo da linguagem científica.

Segundo Norris e Phillips (2003), a leitura do texto científico abrange grande parte do que é considerado fazer ciência, desta forma:

[...] a leitura e escrita não suporta apenas uma relação funcional com respeito à ciência, como simples ferramenta para o armazenamento e transmissão da ciência. Ao contrário, a relação é constitutiva, em que a leitura e a escrita são partes constitutivas da ciência. Relações constitutivas definem necessidades, porque os componentes são elementos essenciais do todo (NORRIS; PHILLIPS, 2003, p. 226).

No espaço formal de aprendizagem, como também no não-formal, torna-se necessário aprender a linguagem científica, pois a sua inserção efetivamente constituída de textos científicos, representações, símbolos, códigos e diferentes tipos de linguagens devam, cada vez mais, estar presente nas aulas e práticas pedagógicas do professor de ciências.

Nos Parâmetros Curriculares em ação (BRASIL, 2002) há ênfase na importância da introdução de conteúdos e elementos linguísticos na sala de aula, pois:

[...] é relevante também considerar as relações com as práticas sociais e produtivas e a inserção do aluno como cidadão em um mundo letrado e simbólico. A produção contemporânea é essencialmente simbólica e o convivio social requer o domínio das linguagens como instrumentos de comunicação e negociação de sentidos. No mundo contemporâneo, marcado por um apelo informativo imediato, a reflexão sobre a linguagem e seus sistemas, que se mostram articulados por múltiplos códigos, e sobre os processos e procedimentos comunicativos, é, mais do que uma necessidade, uma garantia de participação ativa na vida social, a cidadania desejada (BRASIL, 2002, p. 32).

Com os estudos nos últimos anos sobre a importância dos modelos científicos, pautados na compreensão da Natureza da Ciência $(\mathrm{NdC})$ e na utilização de representações teóricas nas explicações dos fenômenos, que podem ser representados na forma de modelos científicos, que apresentam suas potencialidades, como também as suas limitações. $O$ documento produzido pela NationalResearchCouncil (NRC, 2012) faz menção aos modelos no Ensino de Ciências. No documento intitulado "A Framework for K-12 Science Education: Practices, CrosscuttingConcepts, and Core Ideas", notamos a importância de explicações com modelos no Ensino de Ciências, pois contribuem para os estudantes aprenderem: (a) a construir suas próprias explicações de fenômenos usando o conhecimento de teorias cientificamente aceitas e ligando-as a modelos e evidências;(b) a usar evidências científicas primárias ou secundárias e modelos para dar suporte ou refutar uma descrição explicativa para um fenômeno;(c) a propor explicações causais apropriadas para o seu nível de conhecimento científico; (d) a identificar as 
lacunas ou os pontos fracos em descrições explicativas (próprias ou de terceiros) (NRC, 2012, p. 69).

Como afirmam Pozo e Crespo (2009), a natureza altamente abstrata das ciências gera uma dificuldade para o estudante em compreender e analisar as propriedades e transformações da matéria, visto que eles precisam estabelecer conexões entre os conceitos e os fenômenos e, ainda, deparam-se com a necessidade de utilizar uma linguagem simbólica e formalizada junto com modelos de representações analógicos que tem a pretensão de representar aquilo que não é observável. A natureza abstrata da ciência exige dos estudantes que operem no nível formal e não mais no nível operacional (HERRON, 1975), ou seja, o estudante operacional formal começa a pensar em termos do que poderia acontecer, e vislumbra todas as mudanças possíveis. Isto o capacita a raciocinar sem suportes visuais, apenas com as representações. Sendo assim, o aluno necessita se apropriar de conceitos científicos fortemente abstratos, estabelecendo pontes entre esses conceitos e fenômenos, ordenado numa necessidade de se utilizar uma linguagem simbólica e formalizados juntamente com modelos de representações analógicas que possibilitam representar aquilo que não é observável no mundo macroscópico.

Os professores, frequentemente, usam apenas um determinado tipo de representação pictórica, o que limita a experiência dos estudantes com os modelos e, dessa forma, não contribuem para a percepção de que um modelo pode ser parcialmente ou completamente inadequado perante o conhecimento científico vigente (BARNEA; DORI, 2000). Em termos gerais, as habilidades viso-espaciais envolvem a ativação, retenção e/ou manipulação de representações mentais e, portanto, estão estreitamente relacionadas com a memória operacional, sendo o sistema cognitivo responsável pela retenção temporária, bem como pelo processamento da informação durante a realização de atividades cognitivas complexas (BADDELEY, 2012). A utilização de representações no Ensino de Ciências passa a favorecer tarefas que exigem uma série de operações cognitivas de domínio espacial, a exemplo de reconhecer convenções gráficas, bem como manipular informações espaciais fornecidas por uma estrutura molecular, e acompanhar mentalmente algumas restrições com base em conceitos químicos. Desse modo, é provável que a aprendizagem de conceitos científicos envolva determinadas habilidades viso-espaciais na execução de determinadas operações cognitivas (WU; SHAH, 2004).

Habilidade se refere à capacidade do indivíduo operar, eficientemente, na esfera cognitiva, determinados tipos de informação(JUAN-ESPINOSA, 1997; COLOM, 1998). Para Wartha (2013), a visualização no Ensino de Ciências tem sido utilizada para apresentar conceitos aos estudantes presentes nos conteúdos curriculares. Conforme o autor, o termo visualização em Ensino de Ciências significa:

[...] o uso de qualquer tipo de representação não verbal, como por exemplo, fórmulas químicas, diagramas, gráficos, símbolos químicos, representações estruturais, fotografias, imagens, modelos, simulações, animações, softwares interativos, com existência concreta ou virtual, em formato $1 D, 2 D$ ou $3 D$ que permitem uma "interpretação científica". Geralmente os termos recursos visuais, ferramentas visuais, representações visuais, por exemplo, são usadas como sinônimos de visualizações. No nosso entender o termo que 
melhor se ajustaria à visualização seria modelagem ou imaginação, uma vez que o termo visualização refere-se a ver, o que não é o caso para os entes químicos, visto que eles são modelados e/ou imaginados (WARTHA, 2013, p. 78).

Justi (2011) chama atenção sobre as concepções dos professores e futuros professores de ciências, pois apresentam uma visão deformada sobre modelos: a de que os modelos são reprodução ou cópias de alguma coisa. Outros professores admitem nunca terem parado para pensar sobre o assunto, situação que repercute nos processos de ensino e aprendizagem das ciências.

Apesar disso, as experiências com a manipulação de modelos por parte dos estudantes parecem ser cruciais no desenvolvimento das habilidades viso-espaciais, e a ocorrência desse desenvolvimento auxilia os estudantes na compreensão de problemas químicos que envolvem a representação de conceitos no nível submicroscópico, determinantes para a compreensão de uma série de propriedades e reatividade de compostos (WU; SHAH, 2004; GILBERT, 2005; SILVA; RIBEIRO, 2008). Esses autores concordam que a capacidade de visualização espacial no Ensino de Ciências é um ponto fundamental, pois sua aprendizagem envolve habilidades viso-espaciais que dão suporte para realizar determinadas operações cognitivas espacialmente, permitindo a transformação de representações bidimensionais em tridimensionais, usando informações explícitas para estabelecer as devidas relações viso-espaciais. A utilização destas informações para estabelecer a relação viso-espacial com as representações apresentadas na resolução de problemas nos testes e nas avaliações externas são necessárias aos estudantes para obterem um desempenho satisfatório durante seu percurso educacional.

\section{Abordagem Metodológica}

Neste artigo, adotamos uma abordagem quantitativa, para análise e discussão das categorias. A partir da análise estatística descritiva, desenvolvemos as interpretações numéricas acerca da taxa de acerto dos itens, bem como o cruzamento dos dados com as variáveis do questionário socioeconômico.

A amostra selecionada para este estudo foi constituída pelos estudantes que realizaram o Exame Nacional do Ensino Médio no Estado de Sergipe. Os itens selecionados foram referentes ao caderno Azul, que são apresentados com uma estrutura composta de um enunciado, suporte e um comando, possuindo apenas uma afirmativa correta (Figura 1). 
Figura 1: Representação da questão 48 prova Azul de CN

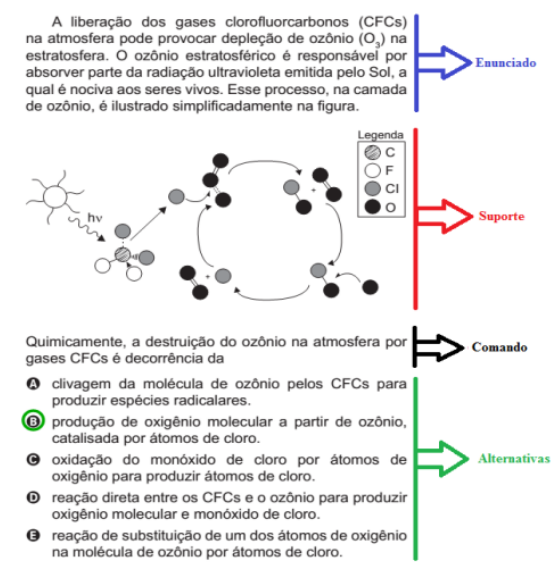

Fonte: Caderno Azul de CN - ENEM 2014.

Para a classificação do desempenho nos itens analisados, consideramos os acertos, segundo a teoria clássica de avaliação. Nesta perspectiva, avaliamos se o suporte representacional nos itens são determinantes para o acerto pelos estudantes que realizaram a prova no Estado de Sergipe. Os dados relacionados aos participantes dos exames, denominados de Microdados, nos anos de 2012, 2013 e 2014, foram obtidos diretamente no endereço eletrônico do INEP (http://portal.inep.gov.br/basica-levantamentos-acessar).

Selecionamos os itens de natureza objetiva para fazerem parte do corpus analítico, tendo como base a análise dos enunciados e suporte, a classificação das competências e habilidades exigidas nas provas, num diálogo entre a teoria e prática. Elaboramos três categorias:

Categoria I - Itens isentos de habilidades de representação: são itens que, de acordo com a matriz de referência, não mencionam necessidade de habilidades sobre uso de representação (equações químicas, fórmula molecular, gráfico, diagramas cartesianos, dentre outros), usadas nas Ciências da Natureza para resolução do problema (Tabela 1).

Tabela 1: Itens selecionados com as Competências e Habilidades

\begin{tabular}{|c|c|c|c|c|c|c|c|c|}
\hline \multicolumn{3}{|c|}{$\begin{array}{c}\text { Categoria suporte } \\
\text { represent acion al - Prova azul - } \\
2012\end{array}$} & \multicolumn{3}{|c|}{$\begin{array}{c}\text { Categoria suporte } \\
\text { represent acional - Prova azul - } \\
2013 \\
\end{array}$} & \multicolumn{3}{|c|}{$\begin{array}{c}\text { Categoria suporte } \\
\text { represent acion al - Prova azul - } \\
2014\end{array}$} \\
\hline Itens & Combetência & Habilidade & Itens & Combetência & Habilid ade & Itens & Combetência & Habilid ade \\
\hline 53 & $\mathrm{C} 5$ & H19 & 46 & $\mathrm{C} 6$ & $\mathrm{H} 20$ & 48 & $\mathrm{C} 3$ & H10 \\
\hline 56 & $\mathrm{C} 4$ & $\mathrm{H} 15$ & 48 & C6 & $\mathrm{H} 22$ & 54 & $\mathrm{C} 1$ & $\mathrm{H} 4$ \\
\hline 59 & $\mathrm{C} 7$ & H26 & 54 & $\mathrm{C} 7$ & $\mathrm{H} 27$ & 55 & $\mathrm{C} 2$ & H6 \\
\hline 67 & $\mathrm{C} 5$ & H18 & 64 & $\mathrm{C} 5$ & H19 & 57 & $\mathrm{C} 2$ & H5 \\
\hline 77 & $\mathrm{C} 2$ & $\mathrm{H} 7$ & 66 & $\mathrm{C} 2$ & $\mathrm{H} 7$ & 62 & C6 & $\mathrm{H} 21$ \\
\hline 82 & $\mathrm{C} 2$ & $\mathrm{H} 8$ & 69 & $\mathrm{C} 7$ & $\mathrm{H} 27$ & 67 & $\mathrm{C} 1$ & $\mathrm{H} 3$ \\
\hline 85 & $\mathrm{C} 4$ & H16 & 73 & $\mathrm{C} 8$ & $\mathrm{H} 29$ & 70 & $\mathrm{C} 7$ & $\mathrm{H} 27$ \\
\hline 89 & $\mathrm{C} 7$ & $\mathrm{H} 15$ & 83 & $\mathrm{C} 2$ & $\mathrm{H} 5$ & 80 & C5 & H19 \\
\hline- & - & - & 85 & C6 & $\mathrm{H} 21$ & 81 & $\mathrm{C} 8$ & $\mathrm{H} 29$ \\
\hline- & - & - & 86 & $\mathrm{C} 5$ & H18 & 82 & C6 & $\mathrm{H} 20$ \\
\hline
\end{tabular}

Fonte: Caderno azul - ENEM - 2012, 2013 e 2014. 
Categoria II - Itens que apresentam habilidade de representação: são itens que, na sua elaboração, foi utilizado o critério de explorar habilidades que envolvem diferentes formas de linguagem científica, representação, símbolos, códigos, nomenclatura e caracterização de materiais ou substâncias, identificando etapas, obtenção ou produção usadas nas Ciências da Natureza (Tabela 2).

Tabela 2: Itens selecionados com base nas Competências e Habilidades

\begin{tabular}{ccccccccc}
\hline \multicolumn{2}{c}{$\begin{array}{c}\text { Categoria suporte } \\
\text { habilid ade-Prova azul - 2012 }\end{array}$} & \multicolumn{2}{c}{$\begin{array}{c}\text { Categoria suporte } \\
\text { habilid ade-Prova azul - 2013 }\end{array}$} & \multicolumn{2}{c}{$\begin{array}{c}\text { Categoria suporte } \\
\text { habilid ade-Prova azul - 2014 }\end{array}$} \\
\hline Itens & Competência & Habilid ade & Itens & Competência & Habilid ade Itens & Competência & Habilid ade \\
\hline 49 & C7 & H24 & 47 & C7 & H24 & 58 & C7 & H24 \\
58 & C7 & H25 & 58 & C7 & H24 & 59 & C7 & H25 \\
60 & C5 & H17 & 68 & C5 & H25 & 64 & C5 & H17 \\
66 & C7 & H25 & 71 & C7 & H25 & 65 & C7 & H24 \\
79 & C7 & H25 & 87 & C5 & H17 & 77 & C7 & H24 \\
90 & C7 & H25 & 90 & C5 & H17 & 78 & C7 & H25 \\
- & - & - & - & - & - & 83 & C5 & H17 \\
- & - & - & - & - & - & 88 & C7 & H25 \\
\hline
\end{tabular}

Fonte: Caderno azul - ENEM - 2012, 2013 e 2014.

Categoria III - Itens sem representação: são itens que o seu enunciado é elaborado predominantemente em forma de linguagem natural ou texto explicativo na solução do problema (Tabela 3).

Tabela 3: Itens selecionados com as Competências e Habilidades

\begin{tabular}{ccccccccc}
\hline $\begin{array}{c}\text { Categoria Suporte } \\
\text { linguagem natural (texto) - } \\
\text { Prova azul - 2012 }\end{array}$ & \multicolumn{2}{c}{$\begin{array}{c}\text { Categoria Suporte } \\
\text { linguagem natural (texto) - } \\
\text { Prova azul - 2013 }\end{array}$} & \multicolumn{2}{c}{$\begin{array}{c}\text { Categoria Suporte } \\
\text { linguagem natural (texto) - } \\
\text { Prova azul - 2014 }\end{array}$} \\
\hline Itens & Competência & Habilidade & Itens & Competência & Habilid ade & Itens & Competência & Habilidade \\
\hline 70 & C7 & H27 & 59 & C3 & H9 & 51 & C3 & H8 \\
76 & C1 & H4 & 61 & C1 & H2 & 56 & C7 & H26 \\
86 & C4 & H15 & 74 & C3 & H8 & 68 & C5 & H19 \\
- & - & - & 77 & C7 & H26 & 75 & C4 & H15 \\
- & - & - & 81 & C1 & H4 & 84 & C1 & H1 \\
- & - & - & 89 & C6 & H21 & 86 & C7 & H24 \\
\hline
\end{tabular}

Fonte: Caderno azul - ENEM - 2012, 2013 e 2014.

Após categorização dos itens e identificação das competências e habilidades avaliadas nas provas aplicadas de 2012 a 2014, realizamos as análises dos dados, com o intuito de estabelecer as relações referentes à problemática investigada nessa pesquisa. Os dados foram filtrados, organizados e analisados com o programa estatístico SPSS (StatisticalPackage for the Social Sciences - versão 23, licença de no 10101151124). 


\section{Resultados}

Considerando os três anos de edições do ENEM utilizados neste estudo, obtivemosuma amostra de 61.112 indivíduos respondentes. Na Tabela 4, apresentamos a variável média das pontuações totais obtidas pelos participantes na prova de CN (Ciências da Natureza) de acordo com o ano e o tipo de escola.

Tabela 4: Média da pontuação total na prova de CN.

\begin{tabular}{cccc}
\hline Área - Ano & Federal & Estadual & Privada \\
\hline $\mathrm{CN}-2012$ & 512,77 & 430,90 & 511,15 \\
$\mathrm{CN}-2013$ & 513,38 & 438,40 & 513,91 \\
$\mathrm{CN}-2014$ & 547,40 & 456,10 & 523,70 \\
\hline Total & $\mathbf{5 2 4 , 5 1}$ & $\mathbf{4 4 1 , 8 0}$ & $\mathbf{5 1 6 , 2 5}$ \\
\hline
\end{tabular}

Fonte: Elaborado pelos autores (2017)

Observamos que os participantes oriundos das escolas federais e privadas obtiveram uma pontuação média semelhante, diferentemente daqueles das escolas estaduais, que obtiveram uma pontuação média consideravelmente mais baixa em todas as edições analisadas. Esse resultado é coerente com a distribuição de escores dos participantes na comparação dos três anos de realização do ENEM. Essas diferenças estão apresentadas nos gráficos de boxplot por dependências (tipo de escola: pública federal, pública estadual e privada) de Ensino Médio (Figura 2).

Figura 2: Box-plot referente à variável pontuação total na prova de CN de acordo com o tipo de escola e o ano.
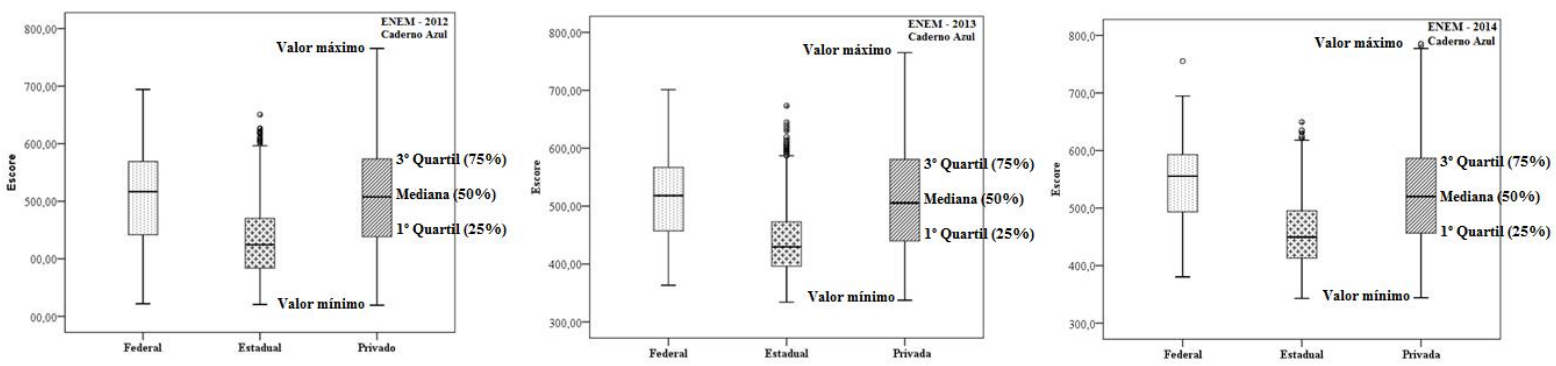

Fonte: Elaborado pelos autores (2017)

Observamos nos gráficos que as escolas Federais e Privadas não apresentam diferença significativa na média das notas, ou seja, os $50 \%$ dos dados estão na mediana dentro do intervalo nos três anos analisados. Em relação à amplitude, no valor máximo, verificamos que as escolas privadas obtiveram notas maiores em comparação às demais modalidades de escolas. Em relação aos dados das escolas estaduais, notamos que há uma diferença significativa: nas três edições do ENEM, as notas estão $75 \%$ abaixo da mediana das demais escolas (federais e privadas).

\section{Análise das categorias com os itens da prova - ENEM - 2012, 2013 e 2014}

Com base nas competências gerais a serem desenvolvidas na área de Ciências da Natureza, destacamos os domínios das representações e comunicação, como meio de verificar se os aspectos representacionais e textuais são determinantes no desempenho escolar, visto 
que nos documentos oficiais o ensino de ciências é apresentado como um dos meios de interpretar o mundo e intervir na realidade.

Ao analisarmos os itens, consideramos a estrutura adotada pelo INEP, mas inserindo mais uma estrutura característicadenominada "suporte do enunciado", que fornece mais um elemento na análise da situação-problema. Apresentamos, nesse momento, os resultados da pesquisa de acordo com as categorias.

Na categoria I, itens isentos de habilidades de representação, obtivemos os seguintes resultados (Tabela 5).

Tabela 5:Taxa de acerto em percentagem nos itens da categoria I

\begin{tabular}{|c|c|c|c|c|c|c|c|c|c|}
\hline \multirow[b]{2}{*}{$\begin{array}{l}\text { Itens- } \\
2012\end{array}$} & \multicolumn{9}{|c|}{ Suporte habilidades } \\
\hline & $\begin{array}{l}\text { (\%) } \\
\text { Acerto }\end{array}$ & Clas. & & $\begin{array}{l}\text { Itens } \\
-2013\end{array}$ & $\begin{array}{l}\text { (\%) } \\
\text { Acerto }\end{array}$ & Clas. & $\begin{array}{c}\text { Itens } \\
-2014\end{array}$ & $\begin{array}{l}\text { (\%) } \\
\text { Acerto }\end{array}$ & Clas. \\
\hline 53 & 26,6 & Baixo & & 46 & 16,1 & Muito Baixo & 8 & 19,5 & Muito Baixo \\
\hline 56 & 37,6 & Baixo & & 48 & 18,7 & Muito Baixo & 4 & 28,7 & Baixo \\
\hline 59 & 24,2 & Baixo & & 54 & 30,9 & Baixo & 5 & 26,5 & Baixo \\
\hline 67 & 29,8 & Baixo & & 4 & 21,8 & Baixo & 7 & 24,5 & Baixo \\
\hline 77 & 25,1 & Baixo & & 6 & 17,3 & Baixo & 2 & 11,6 & Muito Baixo \\
\hline 82 & 21,2 & Baixo & & 9 & 22,7 & Baixo & 7 & 14,0 & Muito Baixo \\
\hline 85 & 33,5 & Baixo & & 3 & 17,8 & Muito Baixo & 0 & 14,7 & Muito Baixo \\
\hline \multirow[t]{3}{*}{89} & 19,3 & $\begin{array}{l}\text { Muito } \\
\text { Baixo }\end{array}$ & & 3 & 14,0 & Muito Baixo & 0 & 36,0 & Baixo \\
\hline & & & - & 5 & 15,3 & Muito Baixo & 1 & 29,3 & Baixo \\
\hline & & & - & 6 & 19,4 & Muito Baixo & 2 & 12,8 & Muito Baixo \\
\hline Prop. & 27,2 & Baixo & & & 19,4 & Muito Baixo & & 23,9 & Baixo \\
\hline
\end{tabular}

Fonte: Elaborado pelos autores (2017).

Os resultados apresentados na Tabela 5 se referem à proporção de acertos nas três edições do ENEM. Notamos resultados não satisfatórios, pois estão classificados na sua maioria com taxas de acertos de desempenho baixo. Para melhor compreender esta questão, optamos por comentar apenas o item que obteve taxa de acerto muito baixo de Ciências da Natureza e apresentar a habilidade correspondente ao item de acordo com a Matriz de Referência (Figura $3)$. 
Figura 3: Itens prova Azul ENEM 2012

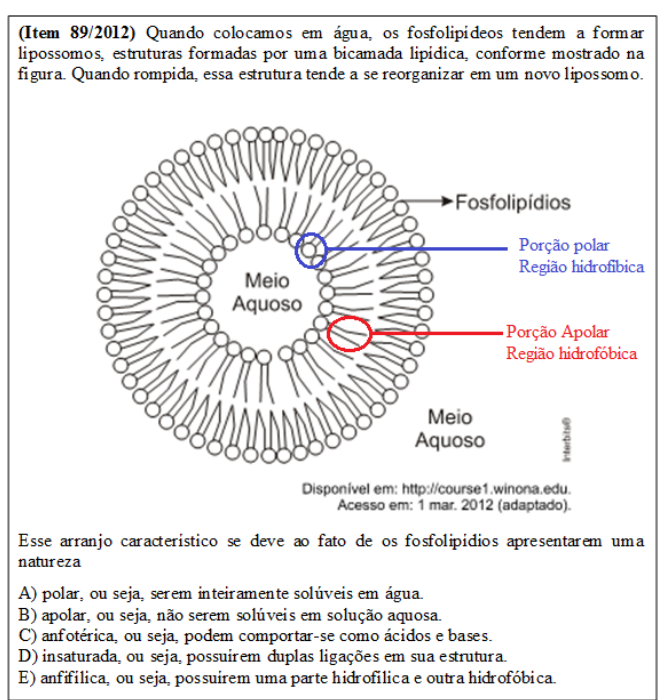

Fonte: Caderno de CN azul do ENEM aplicada em 2012.

A Tabela 6 mostra que a maioria dos participantes não conseguiu interpretar o enunciado e, como consequência, foram confundidos pelos distratores. Um dado interessante é que na questão acima (Figura 3), 27,3\% dos estudantes marcaram o distrator "D". Existe a possibilidade de que os estudantes, ao visualizarem a estrutura da micela e dos fosfolipídios com dois traços, a tenham associado a uma dupla ligação e, portanto, optaram pelo distrator " $D$ ". No entanto, a resposta correta está na letra "E", que explora conceitos científicos, mas que apenas 19,3\% reconheceram como válida. Os aspectos representacionais apresentados no suporte podem ter levado os estudantes a optarem pela alternativa que mais se aproximava do que observavam.

Tabela 6: Percentual de marcação e frequência por alternativas 2012.

\begin{tabular}{llc}
\hline & \multicolumn{2}{c}{ Item $\mathbf{8 9 / 2 0 1 2}$} \\
\hline Alternativa & Freq & $(\%)$ \\
\hline $\mathrm{A}$ & 2.967 & 18,4 \\
$\mathrm{~B}$ & 3.622 & 22,4 \\
$\mathrm{C}$ & 1.835 & 11,4 \\
$\mathrm{D}$ & 4.420 & 27,3 \\
$\mathrm{E}$ & 3.119 & 19,3 \\
Branco & 178 & 1,1 \\
Inválido & 23 & 0,1 \\
\hline Total & 16.164 & 100,0 \\
\hline
\end{tabular}

Fonte: Elaborado pelo autor (2017).

A habilidade H15, isto é, "interpretar modelos e experimentos para explicar fenômenos ou processos biológicos em qualquer nível de organização dos sistemas biológicos", está associada a aspectos representacionais e conceitos científicos que foram utilizados nesse item. Desta forma, imaginamos que o baixo acerto se deve à falta de compreensão conceitual do fenômeno apresentado.

Em relação à categoria com suporte habilidade, os participantes necessitam utilizar, na resolução dos itens, conhecimentos sobre a linguagem científica para a representação e as 
transformações químicas, físicas, biológicas, através de símbolos, fórmulas, convenções e códigos. A competência é a de reconhecer e saber utilizar essa linguagem, sendo capaz de entender e empregar, a partir das informações, a representação simbólica das transformações analisadas. Os resultados estão apresentados na Tabela 7.

Tabela 7: Percentagem de acertos sobre os itens da categoria II.

\begin{tabular}{|c|c|c|c|c|c|c|c|c|}
\hline & \multicolumn{8}{|c|}{ Suporte Habilidade } \\
\hline $\begin{array}{l}\text { Itens- } \\
2012\end{array}$ & $\begin{array}{l}\text { (\%) } \\
\text { Acerto }\end{array}$ & Clas. & $\begin{array}{l}\text { Itens- } \\
2013\end{array}$ & $\begin{array}{l}\text { (\%) } \\
\text { Acerto }\end{array}$ & Clas. & $\begin{array}{c}\text { Itens- } \\
2014\end{array}$ & $\begin{array}{l}\text { (\%) } \\
\text { Acerto }\end{array}$ & Clas. \\
\hline 49 & 17,1 & Muito Baixo & 47 & 27,0 & Baixo & 8 & 0,3 & Baixo \\
\hline 58 & 34,3 & Baixo & 58 & 16,1 & Muito Baixo & 9 & 17,3 & Muito Baixo \\
\hline 60 & 25,3 & Baixo & 68 & 23,7 & Baixo & 4 & 20,3 & Baixo \\
\hline 66 & 17,4 & Muito Baixo & 71 & 20,1 & Baixo & 5 & 17,0 & Muito Baixo \\
\hline 79 & 34,7 & Baixo & 87 & 17,5 & Muito Baixo & 7 & 23,9 & Baixo \\
\hline 90 & 19,2 & Muito Baixo & 90 & 27,7 & Baixo & 8 & 29,9 & Baixo \\
\hline & & - & & & - & 3 & 22,5 & Baixo \\
\hline & & - & & & - & 8 & 24,3 & Baixo \\
\hline Prop. & 24,6 & Baixo & & 20 & Baixo & & 20 & Baixo \\
\hline
\end{tabular}

Fonte: Elaborado pelos autores (2017).

Observamos, mais uma vez, taxa de desempenho baixo e muito baixo nas três edições do exame (Tabela 7). Por exemplo, o item 58 (ENEM-2013) obteve taxas de acerto muito baixo na área de Ciências da Natureza (Figura 4).

Figura 4: Item prova Azul ENEM-2013

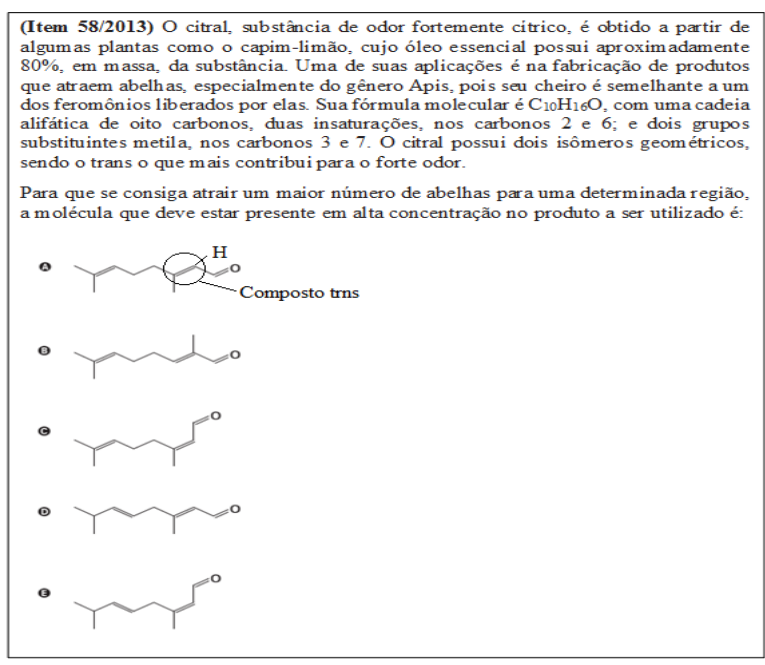

Fonte: Caderno de CN azul do ENEM aplicada em 2013. 
Dentre as alternativas disponíveis, os distratores " $C$ " e " $D$ " foram escolhidos como resposta pela maioria dos alunos, enquanto a afirmação na letra " $A$ " foi assinalada como resposta correta na proporção de 16,1\% (Tabela 7).

Tabela 8: Percentual de marcação e frequência por alternativas 2013.

\begin{tabular}{|c|c|c|c|}
\hline \multicolumn{4}{|c|}{ Item - 58/2013 } \\
\hline Alternative & Freq & & (\%) \\
\hline A & & 3.340 & 6,1 \\
\hline $\mathrm{B}$ & & 4.650 & 2,5 \\
\hline$c$ & & 5.400 & 6,1 \\
\hline D & & 3.831 & 8,5 \\
\hline$E$ & & 3.245 & 5,7 \\
\hline Branco & & 147 & 0,7 \\
\hline Inválido & & 95 & 0,5 \\
\hline Total & & 16.164 & 00,0 \\
\hline
\end{tabular}

Fonte: Elaborado pelos autores (2017).

Percebemos que os participantes não souberam utilizar a habilidade necessária no item 58 , ou seja, a habilidade 24 que faz menção de "utilizar códigos e nomenclatura da química para caracterizar materiais, substâncias ou transformações químicas".

Por fim, a categoria com suporte linguagem natural traz os itens que necessitavam de uma leitura e interpretação dos conhecimentos científicos sobre os fenômenos. Neste contexto, Nigro e Trivelato (2005) enfatizam que o modelo interativo-construtivo de leitura possibilita os estudantes representarem cognitivamente os eventos, os objetos, situações, e construírem ativamente significados ao interagirem com o texto.

Na Tabela 9, mostramos os resultados dos itens classificados como "linguagem natural explicativa" na solução do problema.

Tabela 9: Percentagem de acertos sobre os itens da categoria III.

\begin{tabular}{ccccccccc}
\hline \multicolumn{7}{c}{ Suporte Linguagem Natural } \\
\hline $\begin{array}{c}\text { Itens- } \\
\mathbf{2 0 1 2}\end{array}$ & $\begin{array}{c}(\%) \\
\text { Acerto }\end{array}$ & Clas. & $\begin{array}{c}\text { Itens- } \\
\mathbf{2 0 1 3}\end{array}$ & $\begin{array}{c}(\%) \\
\text { Acerto }\end{array}$ & Clas. & $\begin{array}{c}\text { Itens- } \\
\text { (\%) }\end{array}$ & Clas. \\
\hline 70 & 14,0 & Muito Baixo & 59 & 14,4 & Muito Baixo & 51 & 37,9 & Baixo \\
76 & 23,6 & Baixo & 61 & 26,7 & Baixo & 56 & 25,7 & Baixo \\
86 & 23,8 & Baixo & 74 & 15,8 & Muito Baixo & 68 & 15,1 & Muito Baixo \\
- & - & - & 77 & 21,1 & Baixo & 75 & 35,5 & Baixo \\
- & - & - & 81 & 24,1 & Baixo & 84 & 20,4 & Baixo \\
- & - & - & 89 & 37,6 & Baixo & 86 & 35,8 & Baixo \\
Prop. & $\mathbf{2 0 , 5}$ & Baixo & & $\mathbf{2 4}$ & Baixo & & $\mathbf{2 8 , 4}$ & Baixo \\
\hline
\end{tabular}

Fonte: Elaborado pelos autores (2017).

Observamos que os itens analisados obtiveram um percentual de acertos menor ou igual a 37,9\%, o que classificamos como uma taxa de acerto baixo. Em outros itens o desempenho foi muito baixo, como no item 68/2014 (Figura 5). 
Figura 5: Item prova Azul ENEM-2014.

(Item 68/2014) Uma proposta de dispositivo capaz de indicar a qualidade da gasolina vendida em postos e, consequentemente, evitar fraudes, poderia utilizar o conceito de refração luminosa. Nesse sentido, a gasolina não adulterada, na temperatura ambiente, apresenta razão entre os senos dos raios incidente e refratado igual a 1,4. Desse modo, fazendo incidir of eixe de luz proveniente do ar com um ângulo fixo e maior que zero, qualquer modificação no ângulo do feixe refratado indicará adulteração no combustivel.

A) Mudou de sentido.

B) sofreu reflexão total

C) Atingiu o valor do ângulo limite.

D) Direcionou-se para a superficie de separação.

E) Aproximou-se da normal à superficie de separação.

Fonte: Caderno de CN azul do ENEM aplicada em 2014.

Esse item explora a habilidade $\mathrm{H} 19$, isto é, "avaliar métodos, processos ou procedimentos das Ciências Naturais que contribuam para diagnosticar ou solucionar problemas de ordem social, econômica ou ambiental".

Tabela 10: Percentual de marcação e frequência por alternativas 2014.

\begin{tabular}{ccc}
\hline \multicolumn{3}{c}{ Item - 68/2014 } \\
\hline Alternativa & Freq. & $(\%)$ \\
\hline A & 5.101 & 21,0 \\
B & 5.412 & 22,3 \\
C & 5.326 & 22,0 \\
D & 4.556 & 18,8 \\
E & 3.673 & 15,2 \\
Branco & 116 & 0,5 \\
Inválido & 56 & 0,2 \\
\hline Total & 16.164 & 100,0 \\
\hline
\end{tabular}

Fonte: Elaborado pelos autores (2017).

A partir da Tabela 10, notamos desempenho muito baixo sobre o item, com a alternativa "E" assinalada por $15,2 \%$ dos participantes. Os distratares obtiveram uma maior taxa de marcação, o que indica que os participantes não conseguiram interpretar a situação-problema exposta nem utilizar as leis da física para solucionar o questionamento.

Após a análise destas categorias, concluímos que os aspectos representacionais não são determinantes no desempenho dos participantes, pois observamos percentuais baixos e muito baixos de acertos nos itens, conforme análise estatística descritiva. Fizemos também uma análise dos itens com suporte "linguagem natural" e notamos desempenho muito baixo ou baixo dos participantes. Entendemos que os aspectos da linguagem natural também não são um fator determinante ou que, em razão da proximidade com o erro estatístico (20\%), não é possível realizar qualquer inferência sobre os resultados.

Diante desses resultados, surgiu a necessidade de analisar possíveis influências de características socioeconômicas no desempenho em Ciências da Natureza que podem ser determinantes no desempenho dos participantes. Dentre as variáveis analisadas, destacamos a diferença de desempenho entre sexo, escolaridade dos pais, e renda familiar. 
Os resultados (Gráfico 1) se referem às percentagens sobre a variável socioeconômica e o gênero, o que ajuda a produzir uma visão mais detalhada do desempenho no exame. 0 cruzamento entre as variáveis fornece mais informações para entender o desempenho dos participantes.

Gráfico 1: Desempenho nas categorias em função do sexo nos itens de CN no Enem 2012 a 2014.

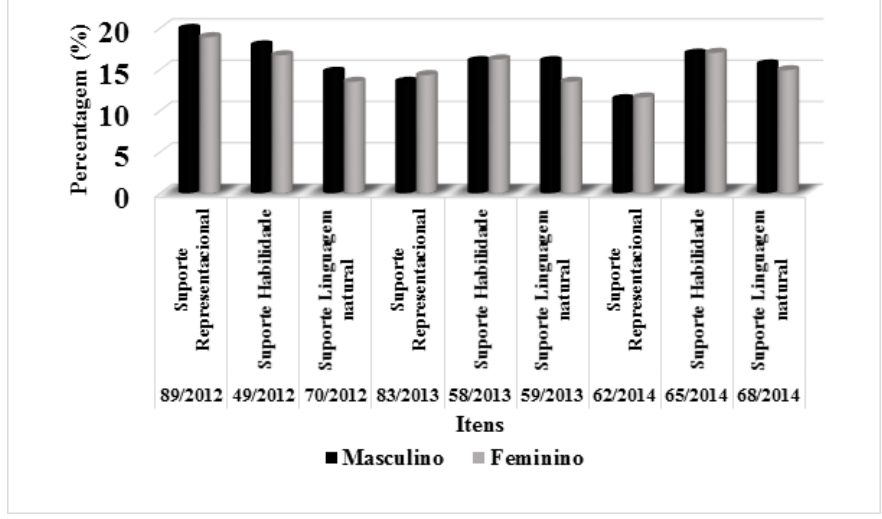

Fonte: Elaborado pelos autores (2017).

Os dados indicam que os sujeitos da pesquisa em ambos os gêneros não demonstram diferenças no desempenho, mesmo que sutis, uma vez que os acertos dos itens estão abaixo de $20 \%$ e seguem bem distribuídos (Gráfico 2).

Consideramos a escolaridade familiar no perfil socioeconômico, pois existe forte relação cultural entre as classes sociais e exclusão escolar. Essa relação pode influenciar as resoluções dos itens. Os resultados no gráfico 2 enfatizam o desempenho nas três categorias.

Gráfico 2: Desempenho nas categorias em função do nível escolar dos pais sobre os itens de CN no Enem 2012 a 2014.

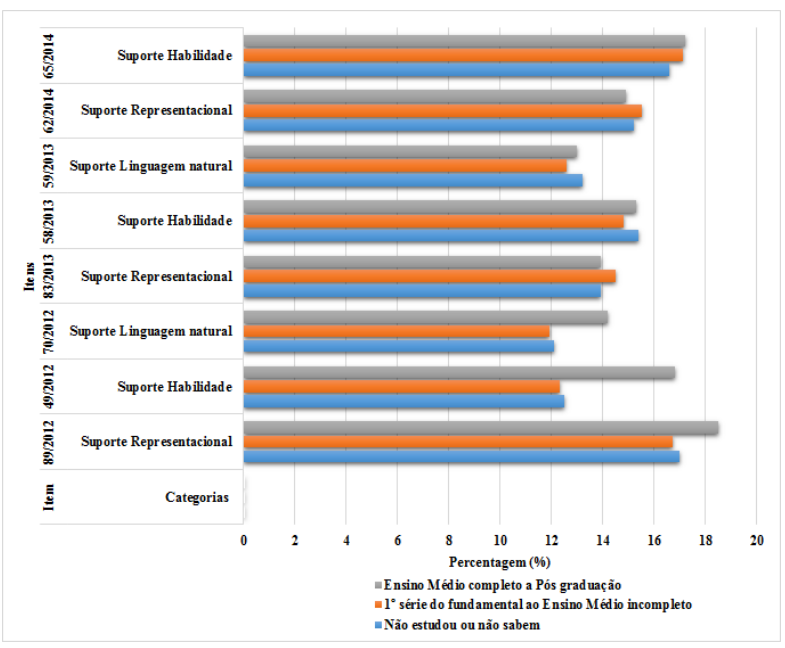

Fonte: Elaborado pelos autores (2016).

Os dados apresentados acima indicam as possibilidades de os participantes acertarem os itens crescem de acordo com a escolaridade familiar, ou seja, quando os participantes possuem pais com pelo menos o Ensino Médio completo e avança até a pós-graduação. No entanto, apesar do gráfico apresentar essa pequena relação com os resultados revelam que ainda permanecem um valor abaixo de $20 \%$ considerado um desempenho muito baixo, partindo 
desta variável não é um fator determinante. Por fim, o gráfico 3 apresenta o cruzamento da variável renda familiar com os itens selecionados.

Gráfico 3: Desempenho na categoria III em função da renda familiar sobre os itens de CN no Enem 2012 a 2014.

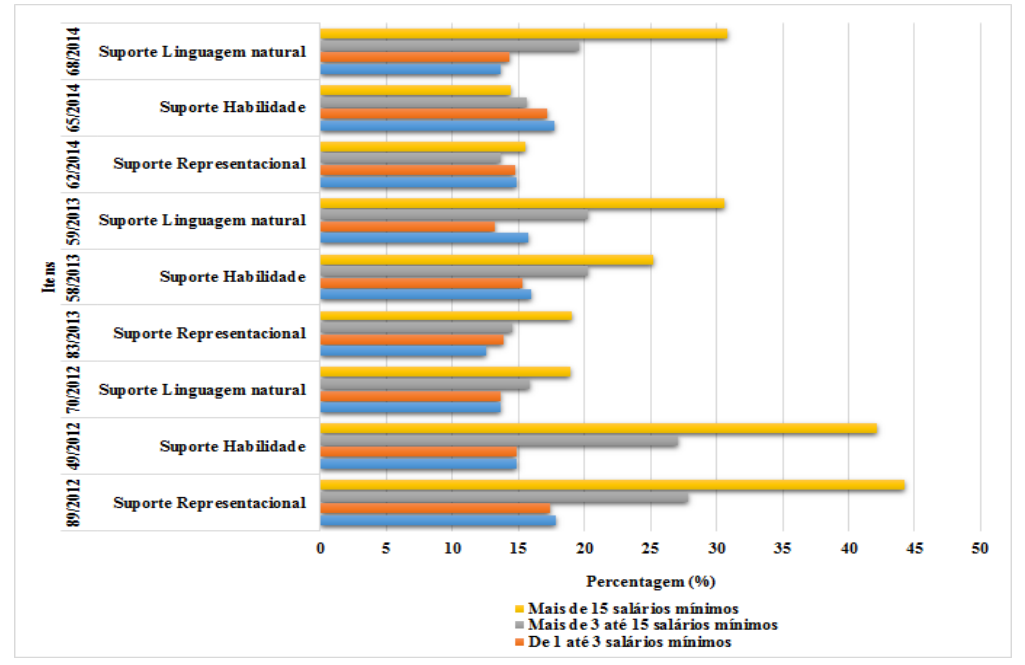

Fonte: Elaborado pelos autores (2017).

$\mathrm{Na}$ análise do gráfico 3, verificamos relação entre aumento de renda familiar e acertos dos itens, o que indica ser a renda um fator determinante no desempenho dos participantes. No entanto, observamos também que, apesar de ser um fator determinantes em relação aos demais considerados neste estudo, a estatística revela uma taxa de acertos abaixo de $45 \%$ de marcação, sendo considerado uma taxa de desempenho médio.

Notamos aumento significativo nas três categorias analisadas (sexo, renda familiar e escolaridade dos país), evidenciado que a partir da renda superior a 3 salários mínimos, com a taxa de acertos aumentando gradativamente, enquanto que a taxa de acerto mais baixo está na faixa de renda familiar inferior a 3 salários mínimos. Nesse contexto, a família é uma variável importante no sucesso acadêmico dos estudantes e é uma das causas, não isoladamente, do fracasso escolar. Tais resultados evidenciam a necessidade de políticas públicas que busquem melhorar a qualidade do ensino, como por exemplo, a adoção da Lei de Cotas, no sentido minimizar essas diferenças entre as classes sociais, bem como maiores investimentos na Educação Básica com incentivos a permanência e a continuidade dos estudos.

\section{CONSIDERAÇÕES}

Os resultados encontrados apontam para uma dificuldade crescente no processo de aprendizagem, do sistema de ensino brasileiro para o ensino de Ciências da Natureza, visto o baixo desempenho dos participantes do exame. Diante dos resultados, concluímos que os aspectos representacionais e/ou habilidade e linguagem natural não permitem destacar de modo concreto diante dos dados estatísticos que são determinantes ou não no desempenho escolar. Nesse sentido, verificamos que os participantes têm um desempenho na maioria dos 
itens classificados como muito baixo ou baixo, mas estatisticamente não é possível afirmar que esse desempenho é devido aos fatores analisados nesse trabalho.

Os resultados também apontam que o perfil socioeconômico dos participantes do Enem de 2012 a 2014 do estado de Sergipe- SE são de pessoas que possuem renda familiar mais baixa normalmente associada a uma menor escolaridade dos pais, influenciando significantemente no desempenho.

Por fim, nas três categorias apresentados, apenas no cruzamento de variáveis renda familiar com os itens levou ao entendimento de que contribui para participantes desenvolverem um desempenho mais significativo no ENEM.

\section{REFERÊNCIAS}

ARAÚJO, R.F.R. Aspectos representacionais e textuais na área de ciências da natureza: um olhar sobre os itens do ENEM. 2017. 152f. Dissertação (Mestrado em Ensino de Ciências e Matemática) - Universidade Federal de Sergipe, São Cristóvão, 2017.

BADDELEY, A. D. Working Memory: Theories, Models, and Controversies. Annual Review of Psychology, v. 63, 1-29. 2012.

BARNEA, N; DORI, Y.J. Computerized molecular modeling the new technology for enhancing model perception among chemistry educators and learners. Chemistry Education: Research and Practice in Europe, 1, 1, 109-120. 2000.

BRASIL. Lei n. 9394/96. Lei de Diretrizes e Bases da Educação Nacional. Brasília, 1996.

BRASIL. Documento básico ENEM 2000. Brasília: MEC/INEP, 2000.

BRASIL.Lei no 10.172, de 9 de janeiro de 2001, aprova o Plano Nacional de Educação e dá outras providências. Brasília: Presidência da República, 2001.

BRASIL.Secretaria de Educação Média e Tecnológica. PCN+ Ensino Médio. Orientações Educacionais Complementares aos Parâmetros Curriculares Nacionais. Ensino Médio. Brasília, 2002.

BRASIL. Instituto Nacional de Estudos e Pesquisas Educacionais Anísio Teixeira. Exame Nacional do Ensino Médio (ENEM):Relatório Pedagógico2009. Brasília, DF, 2009.

BRASIL. Instituto Nacional de Estudos e Pesquisas Educacionais Anísio Teixeira. Exame Nacional do Ensino Médio (ENEM):Textos Teóricos metodológicos 2009. Brasília, DF, 2009.

BRASIL.Lei n. 9394/96. Lei de Diretrizes e Bases da Educação Nacional. Brasília, 1996.

COLOM, R.B.M. Psicología de las Diferencias Individuales. Teoría y Práctica. Madrid: Pirámide. 1998.

FRANCISCO-JUNIOR, W.E. Estratégias de Leitura e Educação Química: Que relações? Química nova na escola. v. 32, n. 4, 2010.

GILBERT, J. K. Visualization in Science Education. Netherlands: Springer, 2005. 
GRANGER, G.G. Filosofia do Estilo. Trad. Scarlett Z. Marton. São Paulo: Perspectiva, Ed. da Universidade de São Paulo, 1974.

JUAN-ESPINOSA, M. Geografia de lainteligencia humana. Madrid: Pirámide. 1997.

JUSTI, R. Modelos e Modelagem no Ensino de Química: um olhar sobre os aspectos essenciais poucos discutidos.In: SANTOS, W. L. P.; MALDANER, O. A. Ensino de Química em Foco. Ijuí: Ed. Unijuí, 2011. p. 209-230.

HERRON, D. Piaget for chemists: explaining what "good" students cannot understand. JournalofChemicalEducation, 52, n.3, p. 156, 1975.

LEMKE, J. L. Aprender a hablar ciência-Lenguaje, aprendizaje y valores. Barcelona: Paidós, 1997. MARQUES, M. O. Escrever é preciso: o princípio da pesquisa.4. Ed. Ijuí: Ed. UNIJUI, 2001, 168p.

N. R. C. A Framework for K-12 Science Education: Practices, Crosscutting Concepts, and Core Ideas. Committee on a Conceptual Framework for New K-12 Science Education Standards. Board on Science Education, Division of Behavioral and Social Sciences and Education. Washington, DC: The National Academies Press, 2012.

NORRIS, Stephen P.; PHILLIPS, Linda M. How literacy in its fundamental sense is central to scientific literacy. Science Education. v. 87, n. 2, p. 224-240, 2003.

NIGRO, R.G.; TRIVELATO, S.L.F. Textos no Ensino-Aprendizagem deCiências: Primeiras Evidências a Favor de um Modelo de Trabalho Baseado em Teorias deLeitura. In: V Encontro Nacional de Pesquisa em Educação em Ciências, Bauru (SP), 2005.

POZO, J. I; CRESPO, M. Á. G. A aprendizagem e o ensino de ciências: do conhecimento cotidiano ao conhecimento científico. Tradução Naila Freitas. 5. ed. Porto Alegre: Artmed, 2009.

SILVA, A; RIBEIRO, N. Modelagem molecular de compostos orgânicos. III Congresso de Pesquisa e Inovação da Rede Norte Nordeste de Educação Tecnológica. Fortaleza, 2008.

SILVA, E. F., RIBAS, M. H.A. Prova do ENEM: O que pensam os professores de matemática? Olhar de Professor, vol. 6, ํo 1, 2003.

WARTHA, E.J. Processos de ensino e aprendizagem de conceitos de química orgânica sob um olhar da semiótica peirceana. 2013. f. 243. Tese (Doutorado ensino de Química) - Faculdade de Educação, Instituto de Física, Instituto de Química e Instituto de Biociências. Universidade de São Paulo, São Paulo, 2013.

WENZEL, J.S. A significação conceitual em química em processo orientado de escrita e reescrita e a ressignificação da prática pedagógica. 2013. Tese (doutorado) - Universidade Regional do Noroeste do Estado do Rio Grande do Sul, ljuí, 2013.

WU, H-K; SHAH, P. Exploring visuospatial thinking in chemistry learning. Science Education, 88(3), 465-492, 2004. 